\title{
ON THE SPECTRAL STABILITY OF SOLITARY WAVE SOLUTIONS OF THE VECTOR NONLINEAR SCHRÖDINGER EQUATION
}

\author{
BERNARD DECONINCK ${ }^{\dagger}$, NATALIE E. SHEILS ${ }^{\dagger}$, NGHIEM V. NGUYEN ${ }^{\ddagger}$, AND \\ RUSHUN TIAN ${ }^{\ddagger}$
}

Abstract. We consider a system of coupled cubic nonlinear Schrödinger (NLS) equations

$$
i \frac{\partial \psi_{j}}{\partial t}=-\frac{\partial^{2} \psi_{j}}{\partial x^{2}}+\psi_{j} \sum_{k=1}^{n} \alpha_{j k}\left|\psi_{k}\right|^{2} \quad j=1,2, \ldots, n,
$$

where the interaction coefficients $\alpha_{j k}$ are real. The spectral stability of solitary wave solutions (both bright and dark) is examined both analytically and numerically. Our results build on preceding work by Nguyen et al. and others. Specifically, we present closed-form solitary wave solutions with trivial and non-trivial phase profiles. Their spectral stability is examined analytically by determining the locus of their essential spectrum. Their full stability spectrum is computed numerically using a large-period limit of Hill's method. We find that all nontrivial-phase solutions are unstable while some trivial-phase solutions are spectrally stable. To our knowledge, this paper presents the first investigation of the stability of the solitary waves of the coupled cubic NLS equation without the restriction that all components $\psi_{j}$ are proportional to sech. bility

Key words. coupled nonlinear Schrödinger, vector nonlinear Schrödinger, solitary waves, sta-

AMS subject classifications. 35Q55, 37K45

1. Introduction. We consider the $n$-component coupled nonlinear Schrödinger (CNLS) system:

$$
i \frac{\partial \psi_{j}}{\partial t}=-\frac{\partial^{2} \psi_{j}}{\partial x^{2}}+\psi_{j} \sum_{k=1}^{n} \alpha_{j k}\left|\psi_{k}\right|^{2}, \quad j=1,2, \ldots, n
$$

where $\psi_{j}(x, t)$ is a complex-valued dimensionless wave function. When $n=1,1.1$. is referred to as the cubic nonlinear Schrödinger (NLS) equation. It has been used extensively to model, among many other applications, waves in deep water [1, 33, 50, propagation in nonlinear optics [26, 30, 49, Bose-Einstein condensates 23, 24, 39, 32], and electron plasma waves [11. The interaction matrix $\boldsymbol{\alpha}=\left(\alpha_{j k}\right)_{j, k=1}^{n}$ contains the information about the nature of the interactions between the different components of the wave functions. Many scalings of the independent and dependent variables that change the effective interaction matrix $\boldsymbol{\alpha}$ are possible. We choose one such that

\footnotetext{
${ }^{\dagger}$ Department of Applied Mathematics, University of Washington, Seattle, WA 98195-2420, USA (bernard@amath.washington.edu, nsheils@amath.washington.edu). The research of these authors was supported by the National Science Foundation under grant NSF-DMS-1008001. Any opinions, findings, and conclusions or recommendations expressed in this material are those of the authors and do not necessarily reflect the views of the funding sources.

‡Department of Mathematics and Statistics, Utah State University, Logan, UT 84322-3900, USA (nghiem.nguyen@usu.edu,rushun.tian@aggiemail.usu.edu)

$\S$ Natalie E. Sheils also acknowledges the National Science Foundation under grant NSF-DGE0718124
} 
diagonal elements are \pm 1 , while the off-diagonal elements are nonzero parameters. This allows for the consideration of the limit to an uncoupled system in which these off-diagonal elements limit to zero. In other words, we regard (1.1) as a perturbation of $n$ scalar NLS equations. Readers are referred to various other works (e.g. [5, 27, 28, 50, 51]) for the derivation of this system in its various application settings.

If system (1.1) is expressed in its original physical dimensions, the matrix $\boldsymbol{\alpha}$ is usually symmetric since the interaction of wave functions with each other is typically symmetric. For example, in applications to Bose-Einstein condensates the governing equations for the interaction of $n \geq 2$ condensates (without the influence of an external potential) are 46 .

$$
i \hbar \frac{\partial \psi_{j}}{\partial t}=-\frac{\hbar^{2}}{2 m_{j}} \frac{\partial^{2} \psi_{j}}{\partial x^{2}}+\sum_{k=1}^{n} \frac{2 \pi \hbar^{2} a_{j k}}{m_{j k}}\left|\psi_{k}\right|^{2} \psi_{j}, \quad j=1, \ldots, n,
$$

where $\psi_{j}(x, t)$ is the wave function for the $j$-th condensate, $m_{j}$ is the atomic mass of the atom species of the $j$-th condensate, $m_{j l}=m_{j} m_{l} /\left(m_{j}+m_{l}\right)$ is the reduced mass corresponding to the atom species of the $j$-th and $l$-th condensates and $a_{j k}=a_{k j}$ is the $s$-wave scattering length between the atomic species $j$ and $k$. Rescaling dependent and independent variables, $m_{j}=2 \hbar, m_{j k}=\hbar, t=2 \pi \tilde{t}, x=2 \sqrt{\pi} \tilde{x}$, and $\psi_{j}=\tilde{\psi}_{j} / \sqrt{a_{j j}}$ we have 1.1 with $\alpha_{j k}=a_{j k} /\left|a_{j j}\right|$. Clearly, $\alpha_{j j}= \pm 1$ but $\alpha_{j k}$ is not necessarily $\alpha_{k j}$. Thus in what follows we cannot impose that the matrix $\alpha$ in 1.1 is symmetric.

The literature discussing the stability of the soliton solutions in the case $n=1$ is extensive, see [7, 31] for instance, as well as references therein. More recently, the literature has focused on the stability of the periodic counterparts of the solitons. This began with Rowlands [43, who found that for the focusing NLS equation $(\alpha>0)$ elliptic solutions are unstable. Gallay and Hărăguş [20, 21, and Hărăguş and Kapitula 25] establish spectral and orbital stability of elliptic solutions for the defocusing NLS equation $(\alpha<0)$. These results are extended by Bottman et al. [7]. Although we only investigate solitary wave solutions, the results for the elliptic solutions are relevant for us, see $\$ 5$

The case of $n=2$ with all elements of $\boldsymbol{\alpha}$ equal is considered in [19. In this case, the system is integrable and more analysis can be done than is undertaken here. In this case, the CNLS system (1.1) is known to possess modulational instabilities experimentally [41, 42] and theoretically [6, 40]. Nguyen et al. [36] consider the system (1.1) with a general power-type nonlinearity and $\alpha_{j k}=\alpha_{k j}$. They prove global existence for small initial data and they establish conditions for finite-time blow-up, which implies instability. Deconinck et al. [16] find two distinct families of solutions which are extensions of the families found for the scalar case, discussed in [8, 9]. We restrict the solutions discussed in [16] to the solitary wave case. These are discussed in detail in the next section. The special case $\alpha_{12}=\alpha_{21}, \alpha_{11}=\alpha_{22}=-1$ of (1.1) with $n=2$ is discussed by Yang and Benney [48. They study the dynamics of both exact and approximate solutions. For the case $\alpha_{12}=\alpha_{21}$ the orbital stability of some solitary wave solutions of (1.1) is established in [35] and [37, see also [3, 4, 38].

This paper discusses the case of stationary solitary wave solutions with both trivial and nontrivial-phase profiles in the case $n=2$. We examine stability for various choices of the interaction matrix $\boldsymbol{\alpha}$ by investigating the locus of the essential spectrum. 
If solutions are found to have a purely imaginary essential spectrum, we use Hill's method to study their spectral stability by computing the discrete spectrum. To our knowledge, this paper presents the first investigation of the stability of the solitary waves of (1.1) without the restriction that all components $\psi_{j}$ are proportional to sech. Specifically we investigate the interaction of bright with dark solitary waves and dark with dark solitary waves. For any case of $\boldsymbol{\alpha}$, the stability study of the nontrivial-phase solutions is new as well.

2. Stationary Solutions. We restrict our attention to stationary solutions of 1.1, i.e. solutions whose time-dependence is of the following form,

$$
\psi_{j}(x, t)=\exp \left(-i \omega_{j} t+i \theta_{j}(x)\right) r_{j}(x),
$$

where $j=1, \ldots, n$, and

$$
\begin{aligned}
\frac{\mathrm{d} \theta_{j}}{\mathrm{~d} x} & =\frac{c_{j}}{r_{j}^{2}(x)}, \\
r_{j}^{2} & =A_{j} \tanh ^{2}(x)+B_{j}, \\
\sum_{l=1}^{n} \alpha_{j l} A_{l} & =2, \\
\omega_{j} & =2+\frac{B_{j}}{A_{j}}+\sum_{l=1}^{n} \alpha_{j l} B_{l}, \\
c_{j}^{2} & =\frac{B_{j}}{A_{j}}\left(A_{j}+B_{j}\right)^{2} .
\end{aligned}
$$

Equations 2.2 are an immediate consequence of substituting the ansatz 2.1 into (1.1). Note that 2.2 gives all solutions of the form (2.1) for (1.1). No additional ansatz is required. In order to uniquely determine the amplitude of the elliptic oscillations $A_{j}$, the matrix $\boldsymbol{\alpha}$ needs to be inverted. The resulting family of solutions has $n$ free parameters $B_{j}, j=1,2, \ldots, n$. If the matrix $\boldsymbol{\alpha}$ is of co-rank $m$ we have an $n+m$-dimensional family of solutions.

If $\mathrm{d} \theta_{j} / \mathrm{d} x=0\left(c_{j}=0\right)$ the solution is referred to as having trivial phase and we choose $\theta_{j}(x)=0$, without loss of generality. With $n=2,2.2$ are written out explicitly as

$$
\begin{aligned}
\frac{\mathrm{d} \theta_{1}}{\mathrm{~d} x} & =\frac{c_{1}}{r_{1}^{2}(x)}, & \frac{\mathrm{d} \theta_{2}}{\mathrm{~d} x} & =\frac{c_{2}}{r_{2}^{2}(x)}, \\
r_{1}^{2} & =A_{1} \tanh ^{2}(x)+B_{1}, & r_{2}^{2} & =A_{2} \tanh ^{2}(x)+B_{2}, \\
2 & =\alpha_{11} A_{1}+\alpha_{12} A_{2}, & 2 & =\alpha_{21} A_{1}+\alpha_{22} A_{2}, \\
c_{1}^{2} & =\frac{B_{1}}{A_{1}}\left(A_{1}+B_{1}\right)^{2}, & c_{2}^{2} & =\frac{B_{2}}{A_{2}}\left(A_{2}+B_{2}\right)^{2} .
\end{aligned}
$$

We have three cases for $\left(\alpha_{11}, \alpha_{22}\right)$ depending on whether the self-interactions are focusing $\left(\alpha_{k k}=-1\right)$ or defocusing $\left(\alpha_{k k}=+1\right)$ : 
1. $\left(\alpha_{11}, \alpha_{22}\right)=(-1,-1)$, i.e. (focusing, focusing),

2. $\left(\alpha_{11}, \alpha_{22}\right)=(-1,1)$, i.e. (focusing, defocusing),

3. $\left(\alpha_{11}, \alpha_{22}\right)=(1,1)$, i.e. (defocusing, defocusing).

The case $\alpha_{11}=1$ and $\alpha_{22}=-1$ can be ignored since it is identical to Case 2 if the indices are switched. Requiring $r_{j}^{2} \geq 0, c_{j}^{2} \geq 0$ for $j=1,2$ restricts our parameter space as displayed for trivial-phase solutions in Figures 2.1, 2.2, 2.3 and for nontrivialphase solutions in Figure 2.4 .

2.1. Trivial-Phase Solutions. Trivial-phase solutions occur for those values of $B_{1}, B_{2}$ for which $c_{1}=c_{2}=0$. Specifically, in the solitary wave case,

$$
\begin{gathered}
B_{j}=0 \Rightarrow \quad \psi_{j}(x, t)=\sqrt{A_{j}} \tanh (x) e^{-i \omega_{j} t} \\
B_{j}=-A_{j} \Rightarrow \quad \psi_{j}(x, t)=\sqrt{-A_{j}} \operatorname{sech}(x) e^{-i \omega_{j} t}
\end{gathered}
$$

We refer to 2.4a as a dark solitary wave while 2.4b is a bright solitary wave.

First, we examine the case when the matrix $\boldsymbol{\alpha}$ has co-rank 1. Then $\alpha_{21}=\alpha_{11}$ and $\alpha_{12}=\alpha_{22}$. We may solve for one of $A_{1}, A_{2}$ in terms of the other. Without loss of generality, let $A_{1}=\left(2-\alpha_{22} A_{2}\right) / \alpha_{11}$.

- For Case 1, $(\operatorname{sech}(x), \operatorname{sech}(x))$ solutions exist for all values of $A_{2} \leq 0$ while $(\tanh (x), \tanh (x))$ solutions are impossible for any value of $A_{2}$. If $A_{2} \geq 0$, $(\operatorname{sech}(x), \tanh (x))$ solutions are possible, while if $A_{2} \leq-2,(\tanh (x), \operatorname{sech}(x))$ solutions exist.

- In Case 2, $(\operatorname{sech}(x), \operatorname{sech}(x))$ solutions exist for all values of $A_{2} \leq 0$ while $(\operatorname{sech}(x), \tanh (x))$ solutions are possible for all values of $A_{2} \geq 0$. If $A_{2} \geq$ 2, $\left(\tanh (x), \tanh (x)\right.$ solutions exist. There are no values of $A_{2}$ for which $(\tanh (x), \operatorname{sech}(x))$ solutions are possible.

- Finally, in Case $3 .(\operatorname{sech}(x), \operatorname{sech}(x))$ and $(\tanh (x), \operatorname{sech}(x))$ solutions exist for all values of $A_{2} \leq 0$. If $0 \leq A_{2} \leq 2,(\tanh (x), \tanh (x))$ solutions are possible. If $A_{2} \geq 0,(\operatorname{sech}(x), \tanh (x))$ solutions exist.

Next, if $\boldsymbol{\alpha}$ is not singular, each of Cases 1, 2, and 3 leads to solutions corresponding to the four combinations of $\operatorname{sech}(x)$ and $\tanh (x)$. The possible values of $\alpha_{12}$ and $\alpha_{21}$ for which solutions exist are shown as shaded regions in Figures 2.1, 2.2. and 2.3 , In Cases 1 and 3 we do not discuss $(\tanh (x), \operatorname{sech}(x))$ solutions because these are the same as $(\operatorname{sech}(x), \tanh (x))$ solutions with the indices switched. These regions are created by requiring 2.3 to be satisfied.

More specifically, in Figures 2.1, $2.1 \mathrm{~b}$, and 2.1 bounding curves are given by the graphs of $\left\{\alpha_{21}=-1\right.$ or $\alpha_{12}=-1$ or $\left.\alpha_{21}=1 / \alpha_{12}>0\right\},\left\{\alpha_{21}=1 / \alpha_{12}>0\right\}$, and $\left\{\alpha_{21}=1 / \alpha_{12}<0\right.$ or $\left.\alpha_{21}=-1\right\}$ respectively. Points are removed from or added to these lines in accordance with the rank-deficient case. Areas shaded in red correspond to unstable solutions where the essential spectrum is not confined to the imaginary axis (i.e., the solution is unstable), whereas areas in blue correspond to solutions that have a purely imaginary essential spectrum, see $\$ 4$. Note that a purely imaginary essential spectrum does not guarantee spectral stability since we have not 
discussed the point spectrum. In Figure 2.1 and the following figures, the notation $\left(\psi_{1}(x), \psi_{2}(x)\right) \sim(f(x), g(x))$ is used to denote that $\psi_{1}(x)$ is proportional to $f(x)$, and $\psi_{2}(x)$ is proportional to $g(x)$.

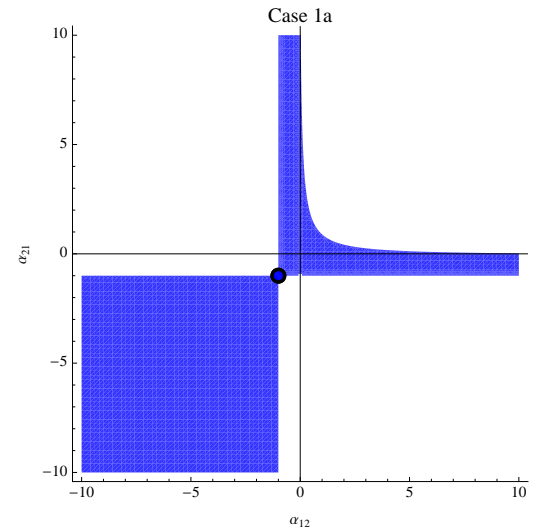

(a)

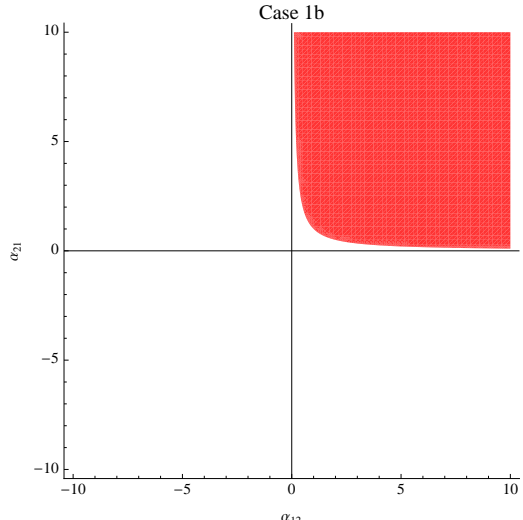

(b)

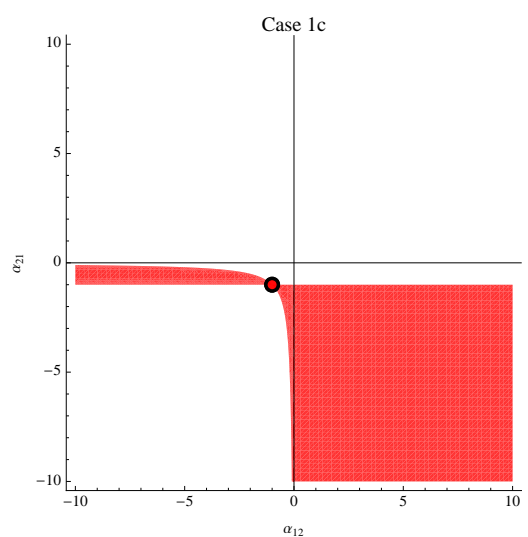

(c)

Fig. 2.1: Ranges of $\left(\alpha_{12}, \alpha_{21}\right)$ for which trivial-phase solutions exist in Case 1 ; $\left(\alpha_{11}, \alpha_{22}\right)=(-1,-1)$. (a) $\left(\psi_{1}(x), \psi_{2}(x)\right) \sim(\operatorname{sech}(x), \operatorname{sech}(x))$, (b) $\left(\psi_{1}(x), \psi_{2}(x)\right) \sim$ $(\tanh (x), \tanh (x))$, and (c) $\left(\psi_{1}(x), \psi_{2}(x)\right) \sim(\operatorname{sech}(x), \tanh (x))$.

Similarly, in Figures $2.2 \mathrm{a}, 2.2 \mathrm{~b}, 2.2 \mathrm{k}$, and $2.2 \mathrm{~d}$ bounding curves are given by the graphs of $\left\{\alpha_{21}=-1 / \alpha_{12}<0\right.$ or $\left.\alpha_{21}=-1\right\},\left\{\alpha_{12}=1\right.$ or $\left.\alpha_{21}=-1 / \alpha_{12}<0\right\},\left\{\alpha_{21}=\right.$ $-1 / \alpha_{12}>0$ or $\alpha_{12}=1$ or $\left.\alpha_{21}=-1\right\}$, and $\left\{\alpha_{21}=-1 / \alpha_{12}>0\right\}$ respectively.

Finally, in Figures $2.3 \mathrm{a}, 2.3 \mathrm{p}$, and 2.3 bounding curves are given by the graphs of $\left\{\alpha_{21}=1 / \alpha_{12}<0\right\},\left\{\alpha_{12}=1\right.$ or $\alpha_{21}=1 / \alpha_{12}<0$ or $\left.\alpha_{21}=1\right\}$, and $\left\{\alpha_{12}=1\right.$ or $\alpha_{21}=$ $\left.1 / \alpha_{12}>0\right\}$ respectively. In addition, Figure $2.3 \mathrm{a}$ includes a point which corresponds to a solution for singular $\boldsymbol{\alpha}$.

2.2. Nontrivial-Phase Solutions. For nontrivial-phase solutions we are unable to make many of the reductions discussed in 2.1 . Once we choose $\alpha_{11}$ and $\alpha_{22}$, the shaded regions of the $\left(\alpha_{12}, \alpha_{21}\right)$-plane do not depend on $B_{1}$ and $B_{2}$ if $B_{1}, B_{2} \geq 0$. 


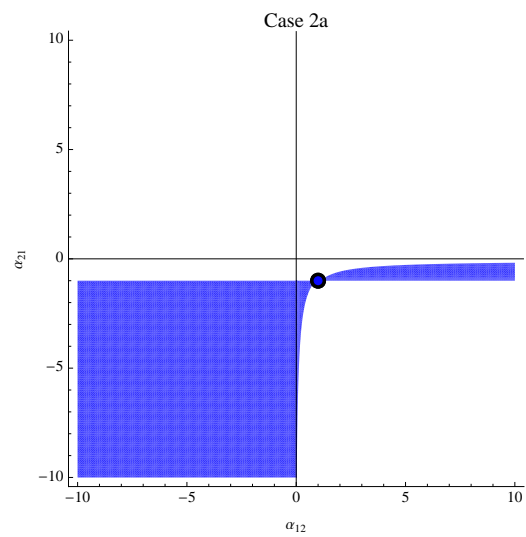

(a)



(c)

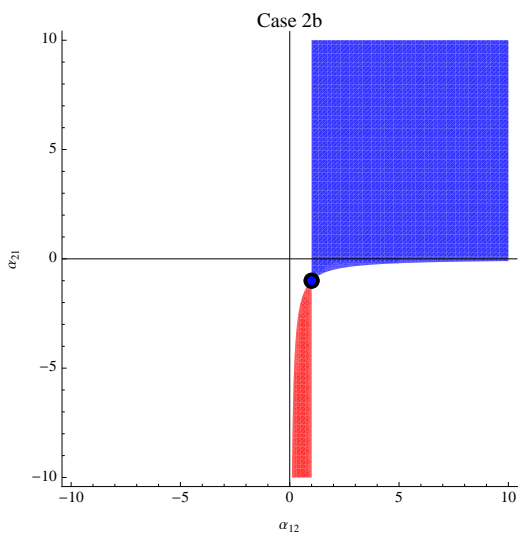

(b)

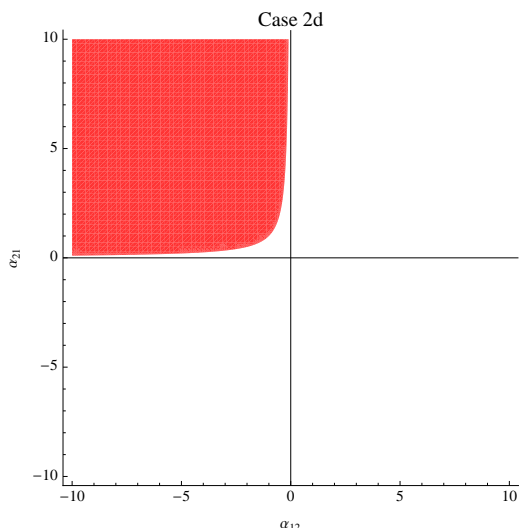

(d)

Fig. 2.2: Ranges of $\left(\alpha_{12}, \alpha_{21}\right)$ for which trivial-phase solutions exist in Case 2 . $\left.\left(\alpha_{11}, \alpha_{22}\right)=(-1,1)\right)$. (a) $\left(\psi_{1}(x), \psi_{2}(x)\right) \sim(\operatorname{sech}(x), \operatorname{sech}(x)),(\mathrm{b})\left(\psi_{1}(x), \psi_{2}(x)\right) \sim$ $(\tanh (x), \tanh (x)),(\mathrm{c})\left(\psi_{1}(x), \psi_{2}(x)\right) \sim(\operatorname{sech}(x), \tanh (x))$, and $(\mathrm{d})\left(\psi_{1}(x), \psi_{2}(x)\right) \sim$ $(\tanh (x), \operatorname{sech}(x))$.

These regions are presented in Figure 2.4. It is of note that for any of the $\left(\alpha_{11}, \alpha_{22}\right)$ cases we can move continuously from nontrivial-phase to trivial-phase solutions by let$\operatorname{ting} B_{j} \rightarrow 0$. We cannot investigate the limit $B_{j} \rightarrow-A_{j}$ since the values of $\alpha_{12}, \alpha_{21}$ necessary to look at this limit are outside of the regions of existence presented in Figure 2.4. The absence of this limit is related to the fact that in the scalar case, nontrivial-phase bright solitons do not exist for focusing NLS whereas for defocusing NLS gray solitons do exist 31 .

In what follows we examine the limit $\left(B_{1}, B_{2}\right) \rightarrow 0$ since in this case nontrivial phase solutions can limit to both stable and unstable trivial-phase solutions.

3. Stability Problem. To examine stability of the exact solutions given in (2.1), we linearize the governing equations 1.1 around these solutions: 


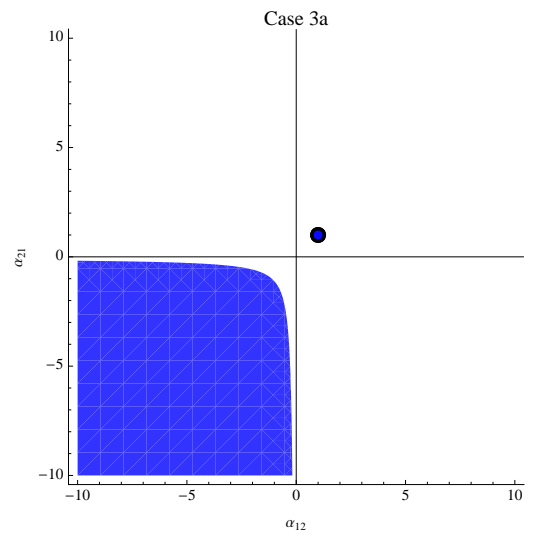

(a)

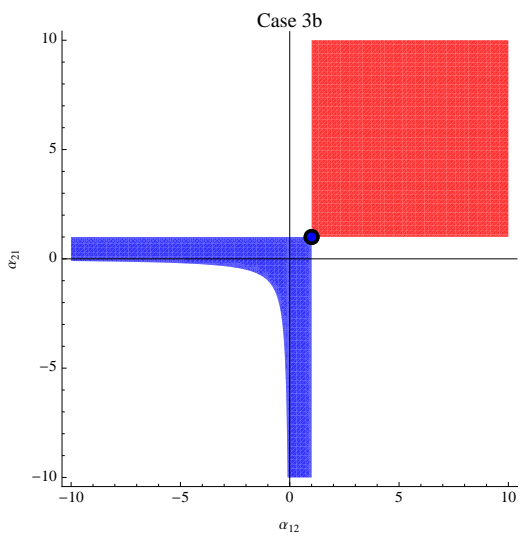

(b)

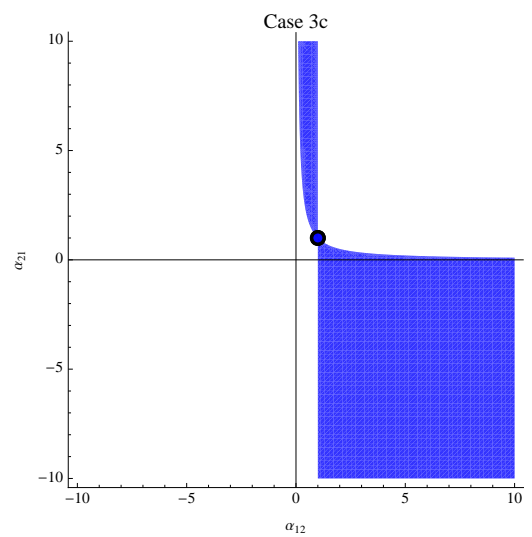

(c)

Fig. 2.3: Ranges of $\left(\alpha_{12}, \alpha_{21}\right)$ for which trivial-phase solutions exist in Case 3 $\left(\alpha_{11}, \alpha_{22}\right)=(1,1)$. (a) $\left(\psi_{1}(x), \psi_{2}(x)\right) \sim(\operatorname{sech}(x), \operatorname{sech}(x))$, (b) $\left(\psi_{1}(x), \psi_{2}(x)\right) \sim$ $(\tanh (x), \tanh (x))$, and (c) $\left(\psi_{1}(x), \psi_{2}(x)\right) \sim(\operatorname{sech}(x), \tanh (x))$.

$$
\psi_{j}(x, t)=e^{i \theta_{j}(x)-i \omega_{j} t}\left(r_{j}(x)+\epsilon \phi_{j}(x, t)+\mathcal{O}\left(\epsilon^{2}\right)\right),
$$

for a small parameter $\epsilon$. Substituting (3.1) into 1.1) and ignoring terms multiplying higher than linear powers in $\epsilon$ gives

$$
\frac{\partial}{\partial t}\left(\begin{array}{c}
u \\
v
\end{array}\right)=J \mathcal{L}\left(\begin{array}{c}
u \\
v
\end{array}\right)=J\left(\begin{array}{cc}
L^{+} & S \\
-S & L^{-}
\end{array}\right)\left(\begin{array}{l}
u \\
v
\end{array}\right)
$$

where $u=\left(u_{1}, \ldots, u_{n}\right)^{T}, v=\left(v_{1}, \ldots, v_{n}\right)^{T}$ and $u_{j}, v_{j}$ are the real and imaginary parts of $\phi_{j}$ respectively. Also, 


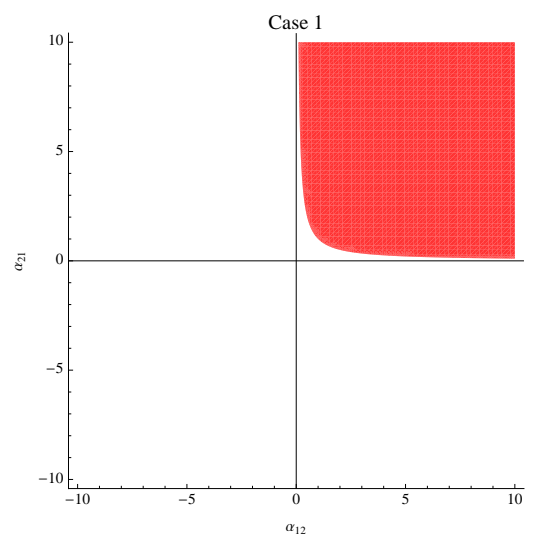

(a)



(b)

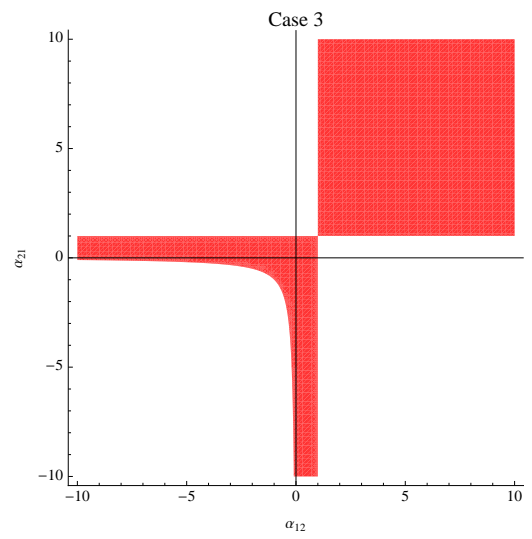

(c)

Fig. 2.4: Ranges of $\left(\alpha_{12}, \alpha_{21}\right)$ for which nontrivial-phase solutions exist given $B_{1}, B_{2} \geq$ 0. (a) $\alpha_{11}=-1, \alpha_{22}=-1$, (b) $\alpha_{11}=-1, \alpha_{22}=1$, and (c) $\alpha_{11}=1, \alpha_{22}=1$.

$$
J=\left(\begin{array}{cc}
0_{n} & 1_{n} \\
-1_{n} & 0_{n}
\end{array}\right)
$$

where $0_{n}$ is the $n \times n$ zero matrix and $1_{n}$ is the $n \times n$ identity matrix. The remaining $n \times n$ matrix operators in $(3.2)$ are given by

$$
\begin{gathered}
\left(L^{+}\right)_{j k}= \begin{cases}-\partial_{x}^{2}+\frac{c_{j}^{2}}{r_{j}^{4}}-\omega_{j}+2 \alpha_{j j} r_{j}^{2}+\sum_{p=1}^{n} \alpha_{j p} r_{p}^{2} & j=k \\
2 \alpha_{j k} r_{j} r_{k} & j \neq k\end{cases} \\
\left(L^{-}\right)_{j k}= \begin{cases}-\partial_{x}^{2}+\frac{c_{j}^{2}}{r_{j}^{4}}-\omega_{j}+\sum_{p=1}^{n} \alpha_{j p} r_{p}^{2} & j=k \\
0 & j \neq k\end{cases}
\end{gathered}
$$




$$
(S)_{j k}= \begin{cases}2 c_{j} \frac{r_{j}^{\prime}-r_{j} \partial_{x}}{r_{j}^{3}} & j=k \\ 0 & j \neq k\end{cases}
$$

where $j, k=1, \ldots, n$.

Since (3.2) is autonomous in $t$, we employ separation of variables and consider solutions of the form $(u(x, t), v(x, t)) \equiv e^{\lambda t}(\hat{u}(x), \hat{v}(x))$. The eigenfunctions $\hat{u}$ and $\hat{v}$ satisfy the spectral problem

$$
\lambda\left(\begin{array}{c}
\hat{u}(x) \\
\hat{v}(x)
\end{array}\right)=J \mathcal{L}\left(\begin{array}{c}
\hat{u}(x) \\
\hat{v}(x)
\end{array}\right)=J\left(\begin{array}{cc}
L^{+} & S \\
-S & L^{-}
\end{array}\right)\left(\begin{array}{c}
\hat{u}(x) \\
\hat{v}(x)
\end{array}\right) .
$$

Since $S$ is anti-symmetric, $\mathcal{L}$ is self adjoint and $(3.6)$ demonstrates the Hamiltonian structure of the problem. In order to show that solutions are spectrally stable, we need to verify that the spectrum of $J \mathcal{L}$ does not intersect the open right half of the complex$\lambda$ plane. That is, there are no $\lambda$ with bounded eigenfunctions satisfying (3.6) such that the real part of $\lambda$ is positive. Since the CNLS equation is Hamiltonian [1, the spectrum of its linearization is symmetric with respect to both the real and the imaginary axis [47. Thus, proving spectral stability of a solution is equivalent to proving that the spectrum of $J \mathcal{L}$ is strictly imaginary. As usual the time-independent spectrum consists of two parts, the essential spectrum and the discrete spectrum.

The analytical investigation of linear stability, beyond the study of the essential spectrum (see $\$ 4$ for nontrivial-phase solutions, is beyond the scope of our methods. However, if all condensate components are described by trivial-phase solutions, more analysis is possible [8, 9, 10. Note that in the trivial-phase case, $S=0_{n}$.

4. The Locus of the Essential Spectrum. The determination of the essential spectrum is an important component for this work. We are able to get analytical results for the essential spectrum of both the trivial and nontrival-phase solutions. If for a particular solution any part of the essential spectrum is in the right-half complex plane, the solution is spectrally unstable and there is no need to examine the stability of the solution numerically unless we not only want to determine stability, but also which modes are the most unstable. To answer this second question, a determination of the entire spectrum is required. If a solution is spectrally unstable due to the location of the essential spectrum we will forego the numerical investigation of the next section.

Definition. A solution for which the essential spectrum of 3.6 is on the imaginary axis is called essentially spectrally stable.

It is clear that solutions that are not essentially spectrally stable are spectrally unstable. The analytical expressions for the essential spectrum provide a means to check the accuracy of the numerical results of the next section.

To compute the essential spectrum we investigate the asymptotic spectral problem determined by letting $|x| \rightarrow \infty$. This results in a constant coefficient problem and we let $(\hat{u}(x), \hat{v}(x)) \equiv e^{i \kappa x}(U, V)$ where $\kappa \in \mathbb{R}$. Equation (3.6) becomes 


$$
\lambda\left(\begin{array}{c}
U \\
V
\end{array}\right)=J \tilde{\mathcal{L}}\left(\begin{array}{c}
U \\
V
\end{array}\right)=J\left(\begin{array}{cc}
\tilde{L}^{+} & \tilde{S} \\
-\tilde{S} & \tilde{L}^{-}
\end{array}\right)\left(\begin{array}{c}
U \\
V
\end{array}\right)
$$

with

$$
\begin{gathered}
\left(\tilde{L}^{+}\right)_{j k}= \begin{cases}\kappa^{2}+\frac{c_{j}^{2}}{r_{j}^{4}}-\omega_{j}+2 \alpha_{j j} r_{j}^{2}+\sum_{p=1}^{n} \alpha_{j p} r_{p}^{2}, & j=k, \\
2 \alpha_{j k} r_{j} r_{k}, & j \neq k,\end{cases} \\
\left(\tilde{L}^{-}\right)_{j k}= \begin{cases}\kappa^{2}+\frac{c_{j}^{2}}{r_{j}^{4}}-\omega_{j}+\sum_{p=1}^{n} \alpha_{j p} r_{p}^{2}, & j=k, \\
0, & j \neq k,\end{cases} \\
(\tilde{S})_{j k}= \begin{cases}2 c_{j} \frac{r_{j}^{\prime}-i \kappa r_{j}}{r_{j}^{3}}, & j=k, \\
0, & j \neq k,\end{cases}
\end{gathered}
$$

for $j, k=1,2, \ldots, n$.

Finding the eigenvalues of the matrix $J \tilde{\mathcal{L}}$ gives a parameterization in terms of $\kappa \in \mathbb{R}$ of the essential spectrum. Eigenvalues with nonzero-real part correspond to unstable modes with a growth rate equal to the absolute value of the real part. In this paper we do not concern ourselves with finding the largest growth rate. Instead we focus on determining whether solutions are spectrally stable.

As mentioned in $\S 1$ we focus on the case $n=2$. In this situation $\tilde{\mathcal{L}}$ is a $4 \times 4$ matrix and the characteristic equation is quartic in $\lambda$. In the trivial-phase case the characteristic equation is biquadratic and we are able to compute explicit parameterizations for the essential spectrum. This is also possible in the nontrivial-phase case but our formulas become extremely unwieldy.

4.1. Trivial-Phase Solutions. For all three cases of $\boldsymbol{\alpha}$, if we are in the trivialphase case such that $B_{j}=-A_{j}$, that is, $\psi_{j}(x) \sim \operatorname{sech}(x)$, 4.1 becomes

$$
\lambda\left(\begin{array}{l}
U \\
V
\end{array}\right)=\left(\begin{array}{cccc}
0 & 0 & 1+\kappa^{2} & 0 \\
0 & 0 & 0 & 1+\kappa^{2} \\
-1-\kappa^{2} & 0 & 0 & 0 \\
0 & -1-\kappa^{2} & 0 & 0
\end{array}\right)\left(\begin{array}{l}
U \\
V
\end{array}\right) .
$$

The eigenvalues are $\lambda= \pm i\left(1+\kappa^{2}\right)$, each with multiplicity two. This results in a double covering of the imaginary axis for values of $|\lambda|>1$, shown in Figure 4.1 . Thus, all $(\operatorname{sech}(x), \operatorname{sech}(x))$ solutions are essentially spectrally stable. It remains to examine their discrete spectrum which is done in the next section.

- For Case 1, that is $\left(\alpha_{11}, \alpha_{22}\right)=(-1,-1)$ the essential spectrum for the other trivial-phase solutions is displayed in Figure 4.2. The analytical formula obtained by solving (4.1) with the proper values of $\alpha_{11}$ and $\alpha_{22}$ implies the 


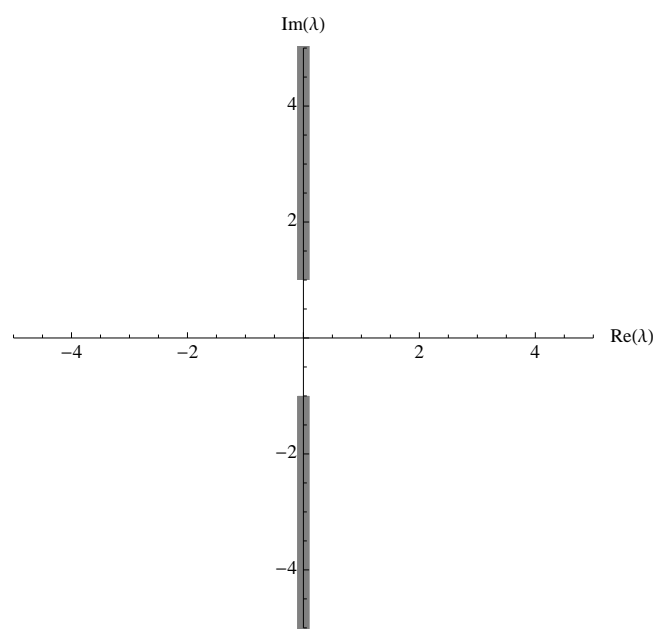

Fig. 4.1: The essential spectrum for any choice of $\alpha_{11}, \alpha_{12}, \alpha_{21}$, and $\alpha_{22}$ with $\left(\psi_{1}(x), \psi_{2}(x)\right) \sim(\operatorname{sech}(x), \operatorname{sech}(x))$.

essential spectrum has nonzero real part. The plots of these analytical formulas presented in Figure 4.2 make it clear that the solutions are unstable in every case shown. The explicit values of $\kappa$ for which the real part of any eigenvalue is positive in the case $\left(\psi_{1}(x), \psi_{2}(x)\right) \sim(\tanh (x), \tanh (x))$ is examined later in this section.



(a)

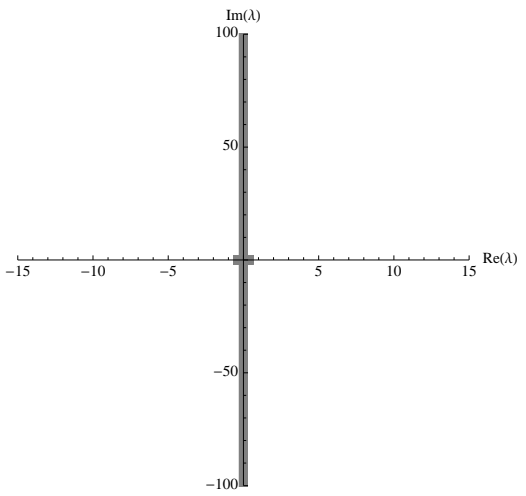

(b)

Fig. 4.2: (a) The essential spectrum for $\alpha_{11}=-1, \alpha_{12}=1.5, \alpha_{21}=2$, and $\alpha_{22}=$ -1 with $\left(\psi_{1}(x), \psi_{2}(x)\right) \sim(\tanh (x), \tanh (x))$. (b) The essential spectrum for $\alpha_{11}=$ $-1, \alpha_{12}=1, \alpha_{21}=-2$, and $\alpha_{22}=-1$ with $\left(\psi_{1}(x), \psi_{2}(x)\right) \sim(\operatorname{sech}(x), \tanh (x))$.

- For Case 2 , that is $\left(\alpha_{11}, \alpha_{22}\right)=(-1,1)$ the essential spectrum for the non$(\operatorname{sech}(x), \operatorname{sech}(x))$ trivial-phase solutions is as given in Figure 4.3 . Notice that for some types of trivial-phase solutions, i.e. $\psi_{1}(x) \sim \tanh (x), \psi_{2}(x) \sim$ $\tanh (x)$, we show two sets of essential spectrum. This corresponds to the two regions of the solution space shown in Figure $2.2 \mathrm{~d}$. 


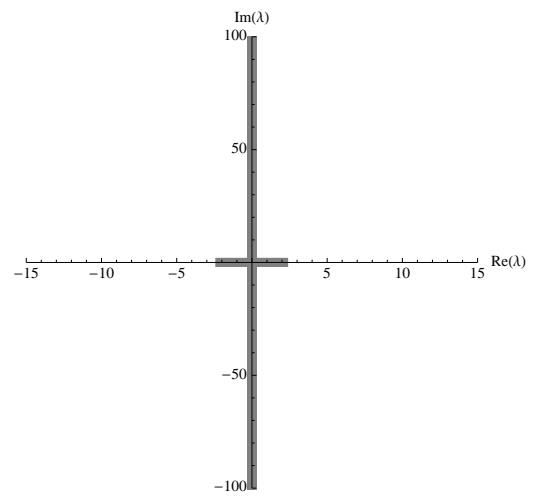

(a)

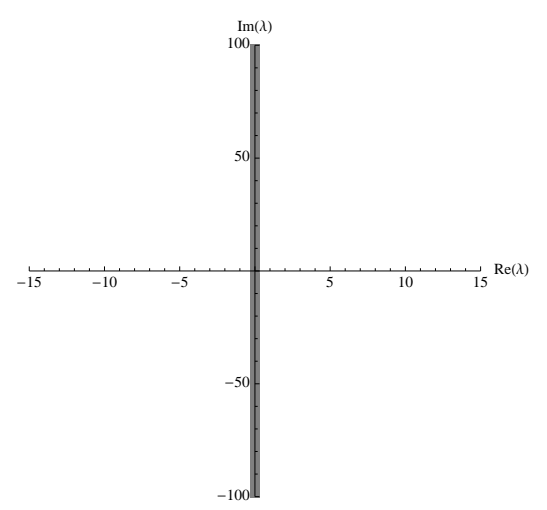

(c)

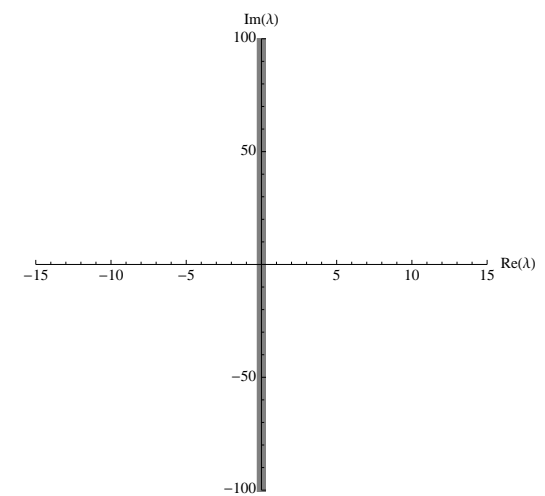

(b)

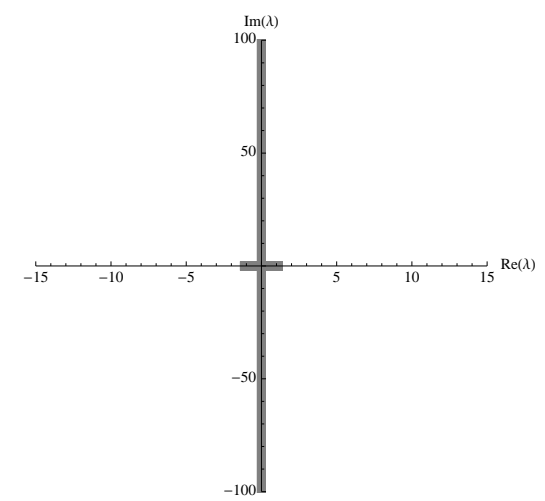

(d)

Fig. 4.3: (a) The essential spectrum for $\alpha_{11}=-1, \alpha_{12}=3, \alpha_{21}=.5$, and $\alpha_{22}=1$ with $\left(\psi_{1}(x), \psi_{2}(x)\right) \sim(\tanh (x), \tanh (x))$. (b) The essential spectrum for $\alpha_{11}=-1, \alpha_{12}=$ $.5, \alpha_{21}=-3$, and $\alpha_{22}=1$ with $\left(\psi_{1}(x), \psi_{2}(x)\right) \sim(\tanh (x), \tanh (x))$. (c) The essential spectrum for $\alpha_{11}=-1, \alpha_{12}=.5, \alpha_{21}=.1$, and $\alpha_{22}=1$ with $\left(\psi_{1}(x), \psi_{2}(x)\right) \sim$ $(\operatorname{sech}(x), \tanh (x))$. (d) The essential spectrum for $\alpha_{11}=-1, \alpha_{12}=-3, \alpha_{21}=1.5$, and $\alpha_{22}=1$ with $\left(\psi_{1}(x), \psi_{2}(x)\right) \sim(\tanh (x), \operatorname{sech}(x))$.

- Finally, for Case 3 that is $\left(\alpha_{11}, \alpha_{22}\right)=(1,1)$ the essential spectrum for the non- $(\operatorname{sech}(x), \operatorname{sech}(x))$ trivial-phase solutions is shown in Figure 4.4. The analytical formula obtained by solving (4.1) with the proper values of $\alpha_{11}$ and $\alpha_{22}$ gives an expression for the essential spectrum. The plots of these analytical formulas presented in Figure 4.4 make it clear when the solutions are unstable. The explicit values of $\kappa$ for which the real part of any eigenvalue is positive in the case $\left(\psi_{1}(x), \psi_{2}(x)\right) \sim(\tanh (x), \tanh (x))$ is examined later in this section.

It is possible to give explicit expressions characterizing $\kappa$ for which the real part of $\lambda$ is positive. For instance in

- Case 11 with $\left(\psi_{1}(x), \psi_{2}(x)\right) \sim(\tanh (x), \tanh (x)),\left(\alpha_{11}=\alpha_{22}=-1, \alpha_{12}>\right.$ 0 , and $\left.\alpha_{21} \alpha_{12}>1\right)$, the essential spectrum is unstable whenever $|\kappa|<$ 


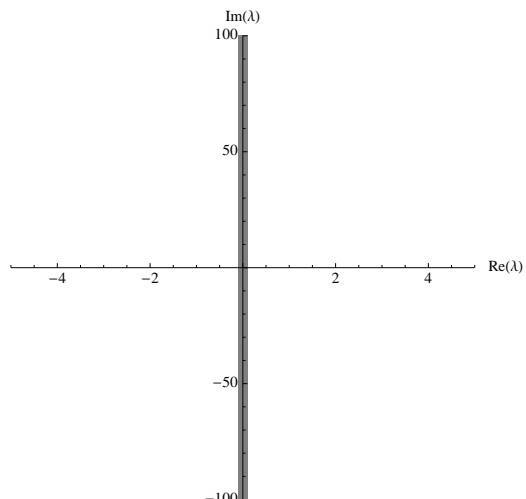

(a)

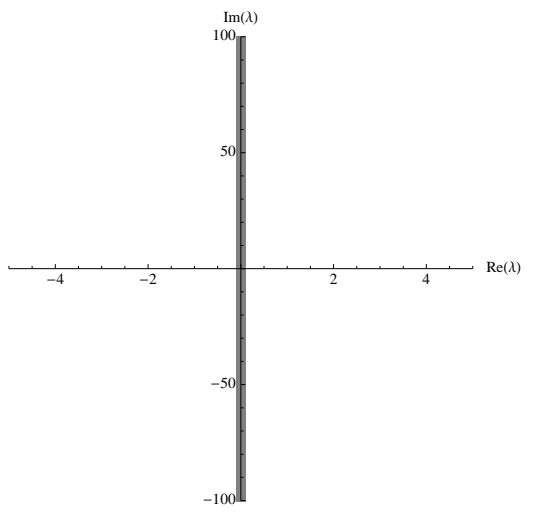

(c)



(b)

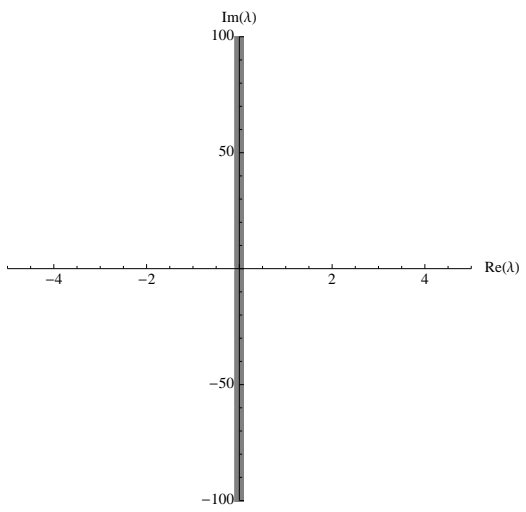

(d)

Fig. 4.4: (a) The essential spectrum for $\alpha_{11}=1, \alpha_{12}=-1.5, \alpha_{21}=.2$, and $\alpha_{22}=$ 1 with $\left(\psi_{1}(x), \psi_{2}(x)\right) \sim(\tanh (x), \tanh (x))$. (b) The essential spectrum for $\alpha_{11}=$ $1, \alpha_{12}=1.5, \alpha_{21}=2$, and $\alpha_{22}=1$ with $\left(\psi_{1}(x), \psi_{2}(x)\right) \sim(\tanh (x), \tanh (x))$. (c) The essential spectrum for $\alpha_{11}=1, \alpha_{12}=.8, \alpha_{21}=3$, and $\alpha_{22}=1$ with $\left(\psi_{1}(x), \psi_{2}(x)\right) \sim$ $(\operatorname{sech}(x), \tanh (x))$. (d) The essential spectrum for $\alpha_{11}=1, \alpha_{12}=2.5, \alpha_{21}=.5$, and $\alpha_{22}=1$ with $\left(\psi_{1}(x), \psi_{2}(x)\right) \sim(\tanh (x), \operatorname{sech}(x))$.

$$
2 \sqrt{\left(\alpha_{12}+1\right) /\left(\alpha_{12}-1\right)} \text { and } \alpha_{12}=\alpha_{21}>1 \text {. }
$$

- Similarly, in Case $2\left(\psi_{1}(x), \psi_{2}(x)\right) \sim(\tanh (x), \tanh (x)),\left(\alpha_{11}=-1, \alpha_{22}=1\right.$ and either $0<\alpha_{12}<1$ and $\alpha_{21}<-1 / \alpha_{12}$ or $\alpha_{12}>1$ and $\left.\alpha_{21}>-1 / \alpha_{12}\right)$, the essential spectrum is unstable whenever $\alpha_{12}=\alpha_{21}>1$ and $|\kappa|>$ $2 \sqrt{\left(\alpha_{12}^{2}-1\right) /\left(\alpha_{12}^{2}+1\right)}$.

- In the final case we consider, Case $3\left(\psi_{1}(x), \psi_{2}(x)\right) \sim(\tanh (x), \tanh (x))$, $\left(\alpha_{11}=\alpha_{22}=1\right.$ and $\left.-1<\alpha_{12}, \alpha_{12} \neq 1\right)$ where the essential spectrum is unstable whenever $\alpha_{12}=\alpha_{21}>1$ and $|\kappa|<2 \sqrt{\left(\alpha_{12}-1\right) /\left(\alpha_{21}-1\right)}$.

Remark. We can examine these cases further and look at the real and imaginary parts of the eigenvalues for all $\kappa \in \mathbb{R}$. For instance, in Case $1 \mathrm{~b}$, if $\alpha_{21}=\alpha_{12}$ the 
eigenvalues are given by

$$
\begin{aligned}
& \lambda_{1,2}= \pm i|\kappa| \sqrt{4+\kappa^{2}}, \\
& \lambda_{3,4}= \pm|\kappa| \sqrt{\frac{4\left(\alpha_{12}+1\right)\left(\alpha_{21}+1\right)+\left(1-\alpha_{12} \alpha_{21}\right) \kappa^{2}}{\alpha_{12} \alpha_{21}-1}} .
\end{aligned}
$$

These eigenvalues form a cross on the imaginary axis similar to that shown in Figure $4.2 \mathrm{a}$. The part on the real axis depends on the values of $\alpha_{12}=\alpha_{21}$. The parameterization of the essential spectrum is not informative because it gives no results on the stability of the solution. We know the essential spectrum is stable (purely imaginary) but we have yet to compute the discrete spectrum which may prove to have nonzero real part which would imply instability.

4.2. Nontrivial-Phase Solutions. As mentioned before, the analytic expressions for the parameterization of the nontrivial-phase solutions in terms of $\kappa$ are too complicated for generic values of $\alpha_{12}, \alpha_{21}, B_{1}$, and $B_{2}$. It is important to note that for both trivial and nontrivial-phase solutions we have an analytic description of the essential spectrum in terms of an implicitly defined quartic curve. In order to create the plots for the more complicated nontrivial-phase case, we compute the roots of the quartic equation numerically to machine precision. In the following figures we present plots of the essential spectrum for a fixed $\left(\alpha_{12}, \alpha_{21}\right)$ while varying $B_{1}$ and $B_{2}$, eventually approaching $B_{1}=B_{2}=0$, the trivial-phase case with $\psi_{j}(x) \sim \tanh (x)$, $j=1,2$. Figures $4.5,4.6,4.7,4.8$ display the essential spectrum for each of the three choices with two possibilities for different values of $\alpha_{12}, \alpha_{21}$ for Case 3

For Cases 1 and 2 and the first choice of $\alpha_{12}, \alpha_{21}$ for Case 3 the essential spectrum always covers the imaginary- $\lambda$ axis and in addition contains a set whose graph has the shape of a figure eight. The figure eight collapses onto the real axis as $B_{1}$ and $B_{2}$ approach zero, eventually limiting to the cross as shown in Figures 4.5, 4.6 and 4.7. This limit is qualitatively the same as that seen in the trivial-phase case in Figures $4.2 \mathrm{a}, 4.3 \mathrm{a}$, and $4.4 \mathrm{a}$. In Figure 4.8 the figure eight collapses onto the imaginary axis and approaches a stable solution as $B_{1}$ and $B_{2}$ go to zero. This mirrors the case shown in Figure $4.4 \mathrm{k}$. In the figures presented in this paper we take the limit $B_{1}, B_{2} \rightarrow 0$ along $B_{1}=B_{2}$. However, taking the limit along any curve with $B_{1}, B_{2} \geq 0$ will also give a series figure eights collapsing to the axis.

Bottman and Deconinck [7] prove stability of trivial- and nontrivial-phase solutions of the scalar defocusing NLS equation. To compare results we choose $\alpha_{22}=\alpha_{11}=1$ and $\alpha_{12}=\alpha_{21}=0$. In this case the eigenvalue problem for the essential spectrum, Equation (4.1) becomes

$$
\lambda\left(\begin{array}{c}
\hat{u} \\
\hat{v}
\end{array}\right)=\left(\begin{array}{cccc}
-i \kappa \sqrt{2 B_{1}} & 0 & \kappa^{2} & 0 \\
0 & -i \kappa \sqrt{2 B_{2}} & 0 & \kappa^{2} \\
-4-2 B_{1}-\kappa^{2} & 0 & -i \kappa \sqrt{2 B_{1}} & 0 \\
0 & -4-B_{2}-\kappa^{2} & 0 & -i \kappa \sqrt{2 B_{2}}
\end{array}\right)\left(\begin{array}{c}
\hat{u} \\
\hat{v}
\end{array}\right) .
$$

The eigenvalues for 4.6 are given by

$$
\lambda_{1,2}=-i \kappa\left(\sqrt{2 B_{1}} \pm \sqrt{4+2 B_{1}+\kappa^{2}}\right),
$$




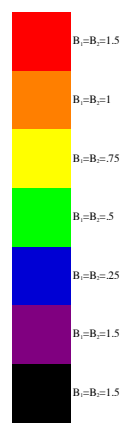

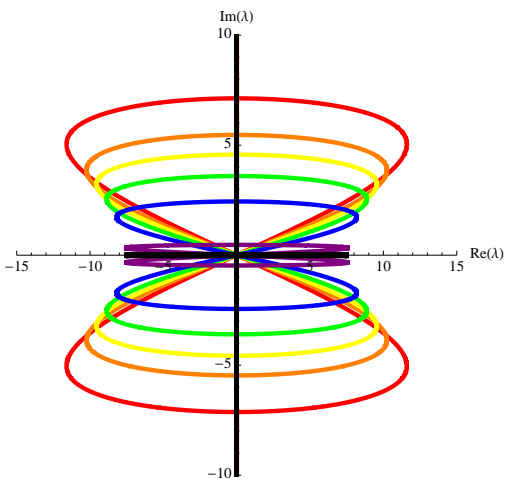

(a)

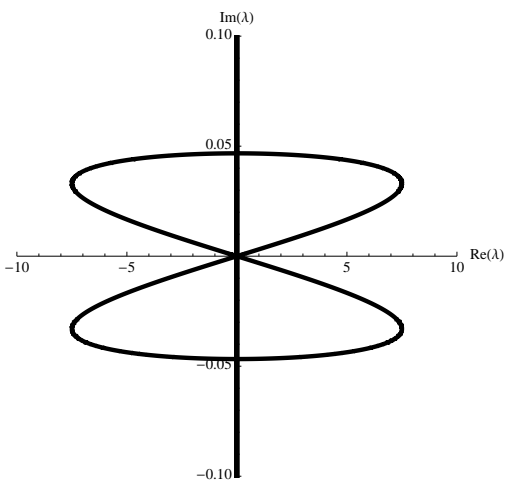

(b)

Fig. 4.5: (a) The essential spectrum of nontrivial-phase solutions with $\alpha_{11}=\alpha_{22}=$ $-1, \alpha_{12}=1.5$, and $\alpha_{21}=2$ for varying values of $B_{1}=B_{2}$ computed analytically. (b) A zoomed in picture for $B_{1}=B_{2}=.0001$.

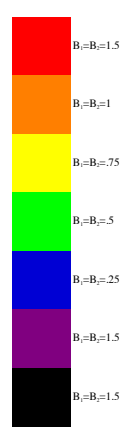



(a)

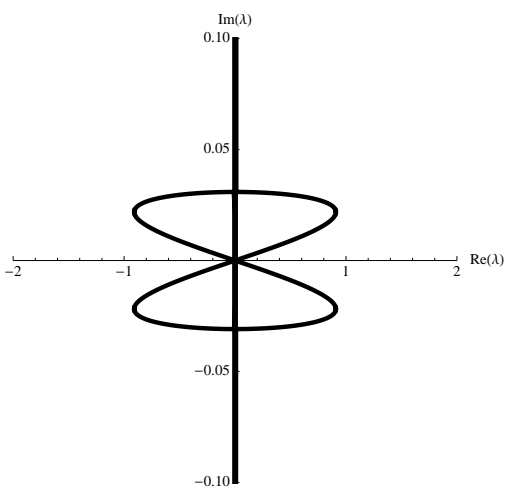

(b)

Fig. 4.6: (a) The essential spectrum of nontrivial-phase solutions with $\alpha_{11}=-1$, $\alpha_{22}=1, \alpha_{12}=1.5$, and $\alpha_{21}=.25$ for varying values of $B_{1}=B_{2}$ computed analytically. (b) A zoomed in picture for $B_{1}=B_{2}=.0001$.

and

$$
\lambda_{3,4}=-i \kappa\left(\sqrt{2 B_{2}} \pm \sqrt{4+2 B_{2}+\kappa^{2}}\right) .
$$

Clearly these are imaginary for $B_{1}, B_{2} \geq 0$. Varying $\kappa$ results in $\lambda$ 's covering the whole of the imaginary axis. This is consistent with the analysis in [7].

Remark. The reader may be familiar with the appearance of a figure eight in the context of the stability analysis of solutions of the NLS equation. Indeed, the stability spectrum a trivial-phase cm-type of the focusing NLS equation consists of the 


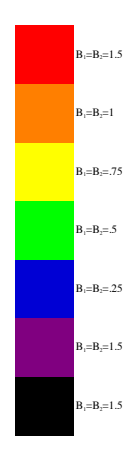



(a)

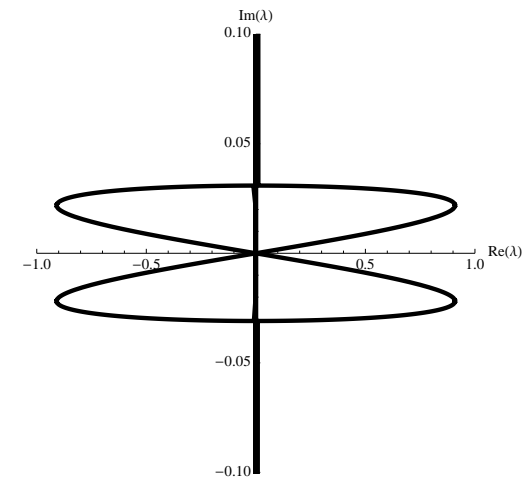

(b)

Fig. 4.7: (a) The essential spectrum of nontrivial-phase solutions with $\alpha_{11}=1, \alpha_{22}=$ $1, \alpha_{12}=2$, and $\alpha_{21}=1.5$ for varying values of $B_{1}=B_{2}$ computed analytically. (b) A zoomed in picture for $B_{1}=B_{2}=.0001$.

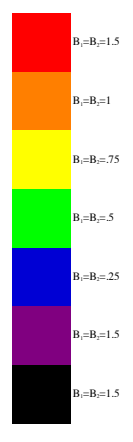

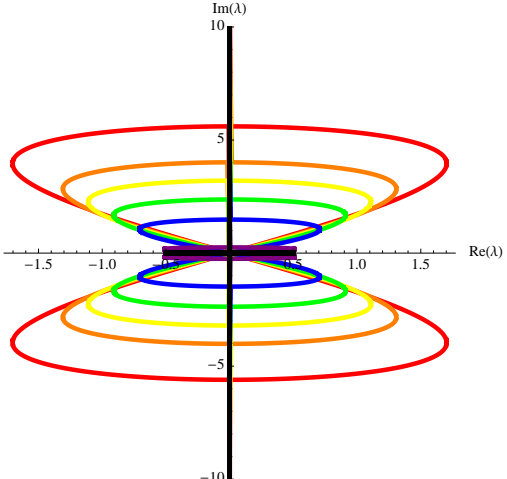

(a)

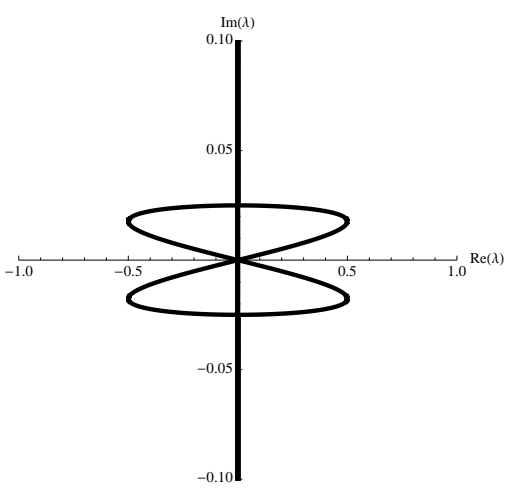

(b)

Fig. 4.8: (a) The essential spectrum of nontrivial-phase solutions with $\alpha_{11}=1, \alpha_{22}=$ $1, \alpha_{12}=-.5$, and $\alpha_{21}=.5$ for varying values of $B_{1}=B_{2}$ computed analytically and (b) a zoomed in picture for $B_{1}=B_{2}=.0001$.

imaginary axis and a figure eight centered at the origin, see [17, 29]. The center of the figure eight is the hallmark of the Benjamin-Feir or modulational instability [43]. The figure eight obtained in our investigations is quite different: our solutions are solitary wave solutions, and the figure eight is the zero set of an explicitly known quartic curve. In addition, the size of the figure eight is significantly larger than that of the trivial-phase cn solution. As a consequence, the growth rates associated with the instabilities found here are larger.

5. The Numerical Computation of the Spectrum. We use Hill's Method as presented in [15. Since the coefficients of $\mathcal{L}$ can be approximated by functions with period $2 L$ we represent them as Fourier series. We recall Floquet's Theorem [2, 13, 18, 
Theorem (Floquet). Consider the linear homogeneous differential equation $y^{\prime}=$ $A(x) y$, for some square matrix $A(x)$ of complex continuous functions such that $A(x+$ $2 L)=A(x)$. Then any fundamental matrix $\Phi(x)$ of this system may be decomposed as $\Phi(x)=\hat{\Phi}(x) e^{R x}$, where $\hat{\Phi}(x+2 L)=\hat{\Phi}(x), \hat{\Phi}(x)$ is nonsingular and $R$ is a constant matrix.

We refer to the eigenvalues of the matrix $R$ as the Floquet exponents. It follows [15] that every bounded solution of $(3.6)$ is of the form

$$
\left(\begin{array}{c}
\hat{u}(x) \\
\hat{v}(x)
\end{array}\right)=e^{i \mu x} \phi(x)
$$

with $\phi(x+2 L)=\phi(x)$ for any fixed $\lambda$ and $\mu \in[0, \pi / L)$. The factor $\exp (i \mu x)$ is referred to as the Floquet multiplier and $i \mu$ is the Floquet exponent (eigenvalue of the matrix $R$ ). In this manuscript we loosely refer to $\mu$ as the Floquet exponent. Writing $\phi(x)$ as a Fourier series such that

$$
\hat{\phi}_{j}=\frac{1}{4 L} \int_{-2 L}^{2 L} \phi(x) e^{\pi i j x /(2 L)} \mathrm{d} x, \quad j \in \mathbb{Z}
$$

Equation (3.6) is equivalent to

$$
\hat{\mathcal{M}}(\mu) \hat{\phi}=\lambda \hat{\phi}
$$

where $\hat{\phi}=\left(\ldots, \hat{\phi}_{-1}, \hat{\phi}_{0}, \hat{\phi}_{1}, \ldots\right)^{T}$ and $\hat{\mathcal{M}}(u)$ is a bi-infinite matrix. It is important to note that no approximations have been introduced to obtain 5.2. In order to obtain a numerical method which approximates the spectrum of (3.6), we choose a cut-off $N$ on the number of Fourier modes of the eigenfunctions $\phi(x)$ which results in a $(2 N+1) \times(2 N+1)$ matrix system

$$
\hat{\mathcal{M}}_{N}(\mu) \hat{\phi}_{N}=\lambda_{N} \hat{\phi}_{N}
$$

where the eigenvalues $\lambda_{N}$ approach $\lambda$ as $N$ approaches infinity.

The convergence of this method as $N \rightarrow \infty$ is proven in [14. This method is spectrally accurate for differential eigenvalue problems with periodic coefficients and an almost-uniform approximation to the entire spectrum is obtained [15]. The numerical component of this method is limited to

1. choosing the size of the matrices to be used, and

2. an eigenvalue solver. 
Hill's method is not intended for linear operators with coefficients that are localized on the whole line as is the case with our problem. In our simulations we approximate the localized function by an elliptic one which converges to it as the elliptic modulus $k$ approaches one [34]. In order to ensure that Hill's method is providing an accurate approximation to the periodic problem, the number of Fourier modes needs to be increased as the period is increased. This is similar to the work done in [12. In using this method of approximating infinite line potentials with periodic potentials with large periods the question arises in what sense the spectrum of such large-period potentials approximates the spectrum of infinite-line potentials. Since the spectrum of the periodic problem is the image of the unit circle in the complex plane as parameterized by the Floquet exponent, Gardner [22] showed that if the period $L$ is sufficiently large, the image of the unit circle is mapped to a simple closed curve containing the spectrum of the infinite line problem. Moreover multiplicities are preserved, implying that no eigenvalues are lost, or no spurious eigenvalues are introduced in the limit.

5.1. Trivial-Phase Solutions. The figures in this section have multiple parts. The blue and red dots correspond to the spectrum computed numerically using Hill's method. Red dots indicate periodic perturbations (Floquet parameter equal to zero) and blue dots represent the full range of the Floquet parameter. The analytic expression for the locus of the essential spectrum computed and discussed in $\$ 4$ is shown in light gray for comparison. All simulations are carried out in python and the formulae used are direct adaptations of those found in [15. For the numerics seen here, we chose the elliptic modulus $k$ to be $1-10^{-16}$. This choice was seen to be sufficiently close to 1 as was discussed above.

In Figure 5.1 in the case of any interaction matrix $\boldsymbol{\alpha}$ for solutions with $\left(\psi_{1}(x), \psi_{2}(x)\right) \sim$ $(\operatorname{sech}(x), \operatorname{sech}(x))$, the solution is stable with essential spectrum lining the imaginary axis for purely imaginary $|\lambda|>1$. The point at the origin is an eigenvalue or member of the discrete spectrum. The numerics agree with the analysis of the essential spectrum in $\$ 4$. This shows that solutions of the form $\left(\psi_{1}(x), \psi_{2}(x)\right) \sim(\operatorname{sech}(x), \operatorname{sech}(x))$ are spectrally stable. In what follows we focus on the remaining cases.

- Case 1 solutions are unstable in every case of $\left(\psi_{1}, \psi_{2}\right)$ and the corresponding $\alpha_{12}, \alpha_{21}$ by virtue of the essential spectrum computed in $\$ 4$.

- Case 2 solutions are spectrally stable if $\left(\psi_{1}(x), \psi_{2}(x)\right) \sim(\operatorname{sech}(x), \tanh (x))$ and for $\alpha_{12}$ and $\alpha_{21}$ in the regions shown in Figure 2.2k. In all other subcases of Case 2, the solutions are unstable. In Figure 5.2 the spectrum for cases which have purely imaginary essential spectrum are shown. As seen in Figure $5.2 \mathrm{a}$, a positive real eigenvalue exists which implies instability. This illustrates the importance of examining both the essential and the discrete spectrum.

- Case 3 solutions are stable if $\left(\psi_{1}(x), \psi_{2}(x)\right) \sim(\operatorname{sech}(x), \tanh (x))$ and for $\alpha_{12}$ and $\alpha_{21}$ in the regions shown in Figure 2.3. If $\left(\psi_{1}(x), \psi_{2}(x)\right) \sim$ $(\tanh (x), \tanh (x))$, solutions have discrete spectrum with nonzero real part and these solutions are unstable. The discrete and essential spectrum are plotted in Figure 5.3 for solutions with purely imaginary essential spectrum. As in the previous case, a real positive eigenvalue exists in the $(\tanh (x), \tanh (x))$ case as seen in Figure 5.3 . 


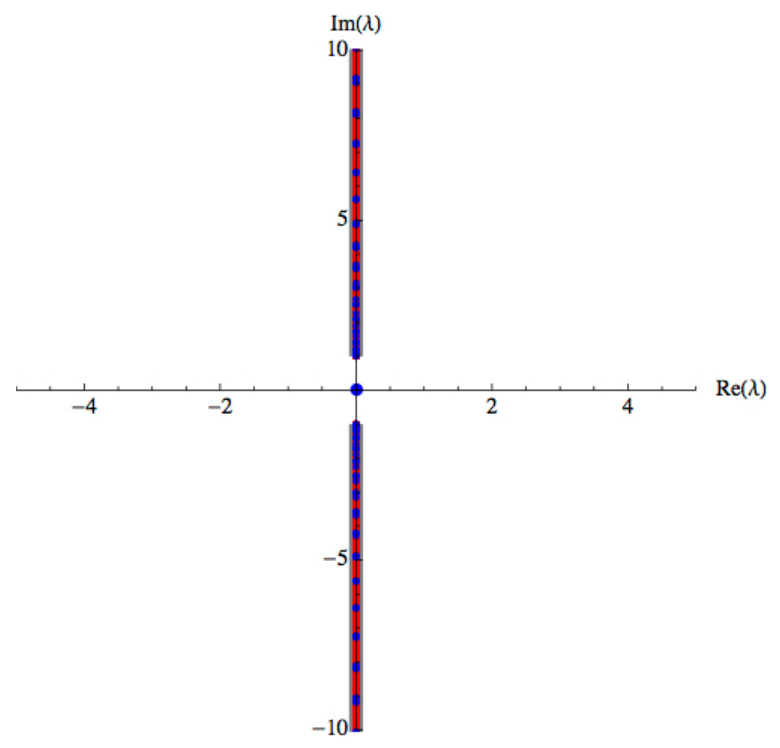

Fig. 5.1: The stability spectrum for any choice of $\alpha_{11}, \alpha_{12}, \alpha_{21}$, and $\alpha_{22}$ with $\left(\psi_{1}(x), \psi_{2}(x)\right) \sim(\operatorname{sech}(x), \operatorname{sech}(x))$.

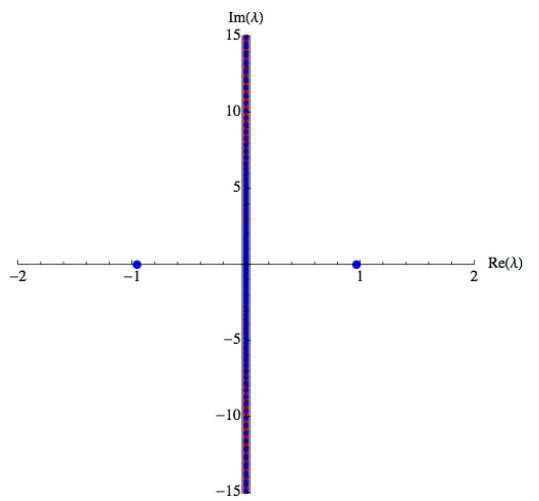

(a)



(b)

Fig. 5.2: (a) The stability spectrum for $\alpha_{11}=-1, \alpha_{12}=.5, \alpha_{21}=-3$, and $\alpha_{22}=1$ with $\left(\psi_{1}(x), \psi_{2}(x)\right) \sim(\tanh (x), \tanh (x))$. (b) The spectrum for $\alpha_{11}=-1, \alpha_{12}=$ $.5, \alpha_{21}=.1$, and $\alpha_{22}=1$ with $\left(\psi_{1}(x), \psi_{2}(x)\right) \sim(\operatorname{sech}(x), \tanh (x))$.

6. Summary. We have thoroughly investigated all cases of the two-component coupled NLS system. Solutions that are spectrally stable are presented in Table 6.1 where $\alpha_{12}$ and $\alpha_{21}$ are chosen to be in the proper ranges as discussed in $\$ 2$. All other solutions, including all nontrival-phase solutions, are spectrally unstable. For the case $\alpha_{12}=\alpha_{21}$, the first four rows of Table 6.1 are consistent with results presented in [3, 4, 35, 37, 38]. 


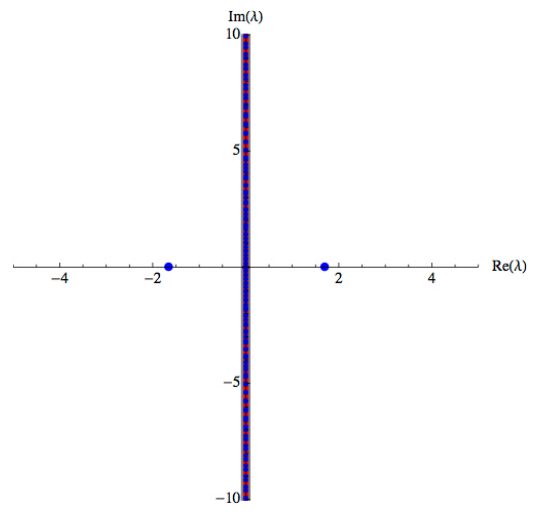

(a)

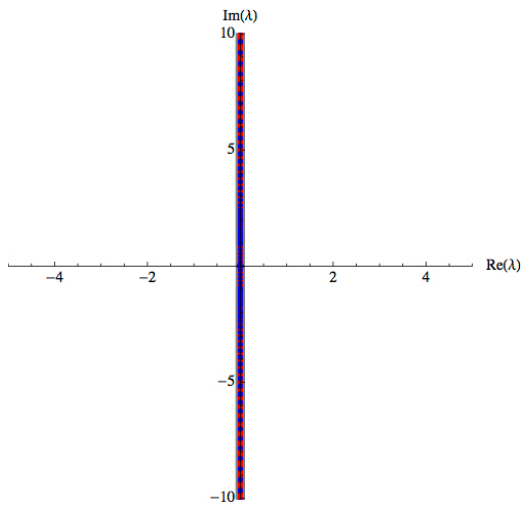

(b)

Fig. 5.3: (a) The stability spectrum for $\alpha_{11}=1, \alpha_{12}=-1.5, \alpha_{21}=.2$, and $\alpha_{22}=$ 1 with $\left(\psi_{1}(x), \psi_{2}(x)\right) \sim(\tanh (x), \tanh (x))$. (b) The stability spectrum for $\alpha_{11}=$ $1, \alpha_{12}=.8, \alpha_{21}=3$, and $\alpha_{22}=1$ with $\left(\psi_{1}(x), \psi_{2}(x)\right) \sim(\operatorname{sech}(x), \tanh (x))$.

\begin{tabular}{|c|c|c|c|}
\hline$\alpha_{11}$ & $\alpha_{22}$ & $\psi_{1}(x)$ & $\psi_{2}(x)$ \\
\hline \hline 1 & 1 & $\operatorname{sech}(x)$ & $\operatorname{sech}(x)$ \\
\hline 1 & -1 & $\operatorname{sech}(x)$ & $\operatorname{sech}(x)$ \\
\hline-1 & 1 & $\operatorname{sech}(x)$ & $\operatorname{sech}(x)$ \\
\hline-1 & -1 & $\operatorname{sech}(x)$ & $\operatorname{sech}(x)$ \\
\hline-1 & 1 & $\operatorname{sech}(x)$ & $\tanh (x)$ \\
\hline 1 & 1 & $\operatorname{sech}(x)$ & $\tanh (x)$ \\
\hline
\end{tabular}

Table 6.1: Solutions we found to be spectrally stable with $\alpha_{12}$ and $\alpha_{21}$ appropriately chosen as discussed in $\$ 2$.

\section{REFERENCES}

[1] Ablowitz, M. J. And Segur, H., Solitons and the Inverse Scattering Transform, SIAM, Philadelphia, PA, 1981.

[2] Amann, H., Ordinary differential equations, de Gruyter Studies in Mathematics, vol 13. Walter de Gruyter \& Co. Berlin, 1990.

[3] Bartsch, T. And Wang, Z.Q., Note on ground states of nonlinear Schrödinger systems, J. Part. Diff. Eqs., 19 (2006), pp. 200-207.

[4] Bartsch, T., Wang, Z.Q., And Wei, J., Bound states for a coupled Schrödinger system, J. Fixed Point Theory Appl., 2 (2007), pp. 353-367.

[5] Benney, D. J., And Newell, A.C., The propagation of nonlinear wave envelopes, J. Math. Phys., 46 (1967), pp. 133-139.

[6] Blow, K. J., Doran, N. J., And Wood, D., Polarization instabilities for solitons in birefringent fibers, Opt. Lett. 12 (1987), pp. 202.

[7] Bottman, N., Deconinck, B., and Nivala, M., Elliptic solutions of the defocusing NLS equation are stable, J. Phys. A 44 (2011), 285201.

[8] Bronski, J. C., Carr L., Carretero-Gonzalez, R., Deconinck, B., Kutz, J. N., And Promislow, K., Stability of Attractive Bose-Einstein Condensates in a Periodic Potential, Phys. Rev. E., 64 (2001), 056615.

[9] Bronski, J. C., Carr, L., Deconinck, B., Kutz, J. N., and Promislow, K., Stability of Repulsive Bose-Einstein Condensates in a Periodic Potential, Phys. Rev. E., 63 (2001), 
036612 .

[10] Bronski, J. C., Carr, L., Deconinck, B., and Kutz, J. N., Bose-Einstein condensates in standing waves: the cubic nonlinear Schrödinger equation with a periodic potential, Phys. Rev. Lett. 86 (2001), pp. 1402-1405.

[11] Chen, Francis F., Introduction to Plasma Physics and Controlled Fusion, 2nd edn. Springer, New York, NY, 1984.

[12] Chen, M., Curtis, C., Deconinck, B., Lee, C., And Nguyen, N., Spectral stability of stationary solutions of a Boussinesq system describing long waves in dispersive media, SIAM J. Appl. Dynam. Sys. 9 (2010), pp. 999-1018.

[13] Coddington, E. A. And Levinson, N., Theory of Ordinary differential equations, McGrawHill Book Company, New York, NY, 1955.

[14] Curtis, C. And Deconinck, B., On the convergence of Hill's method, Math. of Comp. 79 (2010), pp. 169-187.

[15] Deconinck, B. And Kutz, J. N., Computing spectra of linear operators using the FloquetFourier-Hill method, J. Comput. Phys., 219 (2006), pp. 296-321.

[16] Deconinck, B., Kutz, J. N., Patterson, M. S., and Warner, B. W., Dynamics of Periodic multi-component Bose-Einstein condensates, J. Phys. A, 36 (2003), pp. 97-103.

[17] Deconinck, B. And Oliveras, K., The instability of periodic surface gravity waves, J. Fluid Mech. 675 (2011), pp. 141-167.

[18] Floquet, G., Sur les équations différentielles linéaires á coefficients périodiques, Ann. École norm. Ser. 2, 12 (1883) pp. 47-89.

[19] Forest, M. G., Mclaughlin, D. W., Muraki, D. J., and Wright, O. C., Nonfocusing instabilities in Coupled, Integrable Nonlinear Schrödinger PDEs, J. Nonlinear Sci. 10 (2000), pp. 291-331.

[20] Gallay, T. AND HĂRĂGuş, M., Orbital stability of periodic waves for the nonlinear Schrödinger equation, J. Dynam. Differ. Equ. 19 (2007), pp. 825-865.

[21] Gallay, T. AND HĂRĂGuş, M., Stability of small periodic waves for the nonlinear Schrödinger equation, J. Differ. Equ. 234 (2007), pp. 544-581.

[22] Gardner, R. A., Spectral Analysis of Long Wavelength periodic waves and applications, J. fur die Reine und Angewandte Mathematik. 491 (1997), pp. 149-181.

[23] Gross, E. P., Structure of a quantized vortex in boson systems, Nuevo Cimento, 20: 45477, 1961.

[24] Hall, D. S., Matthews, M.R., Ensher, J.R., Wiemann, C.E., and Cornell, E.A., The Dynamics of Component Separation in a Binary Mixture of Bose-Einstein Condensates, Phys. Rev. Lett., 81 (1998), 1539.

[25] HăRĂGuS, M. AND Kapitula, T., On the spectra of periodic waves for infinite-dimensional Hamiltonian systems, Phys. D, 237 (2008), pp. 2649-2671.

[26] Hasegawa, A., Optical Solitons in Fibers, Springer, New York, NY, 1990.

[27] Hasegawa, A. And TAPpert, F., Transmission of stationary nonlinear optical pulses in dispersive dielectric fibers I. Anomalous dispersion, Appl. Phys. Lett., 23 (1973), pp. 142-144.

[28] Hasegawa, A. And TAPpert, F., Transmission of stationary nonlinear optical pulses in dispersive dielectric fibers II. Normal dispersion, Appl. Phys. Lett., 23 (1973), pp. 171-172.

[29] Ivey, T. And Lafortune, S., Spectral stability analysis for periodic traveling wave solutions of NLS and CGL perturbations, Phys. D, 237 (2008), pp. 1750-1772.

[30] Kivshar, Y. S. and Agrawal, G., Optical Solitons: From Fibers to Photonic Crystals, Academic Press, San Diego, CA, 2003.

[31] Kivshar, Y. S. and Luther-Davies, B., Dark optical solitons: physics and applications, Phys. Rep. 298 (1998), pp. 81-197.

[32] Myatt, C. J., Burt, E. A., Ghrist, R. W, Cornell, E. A., and Wieman, C. E., Production of Two Overlapping Bose-Einstein Condensates by Sympathetic Cooling, Phys. Rev. Lett., 78 (1997), pp. 586.

[33] Newell, A. C., Solitons in Mathematics and Physics, SIAM: Philadelphia, PA: 1985.

[34] NIST Digital Library of Mathematical Functions. http://dlmf .nist.gov, Release 1.0.5 of 2012$10-01$.

[35] NGUyen, N., On the orbital stability of solitary waves for the 2-coupled nonlinear Schrödinger system, Comm. Math Sci. 9.4 (2011), pp. 997-1012.

[36] Nguyen, N., Tian, R., Deconinck, B., and Sheils, N. E., Global Existence for a Coupled System of Schrödinger Equations with Power Type Nonlinearity, Jour. Math. Phys. 54 (2013), 013701.

[37] NGUYen, N. AND WANG, Z., Orbital stability of solitary waves for a nonlinear Schrödinger system, Adv. Diff. Eqns. 16 (2011), pp. 977-1000 .

[38] Онта, M., Stability of solitary waves for coupled nonlinear Schrödinger equations, Nonlinear 
Anal. Theory, Methods \& Appl., 26 (1996), pp. 933-939.

[39] Pitaevskit, L. P., Vortex lines in an imperfect Bose gas, Sov. Phys. JETP 13 (1961), pp. 451-454.

[40] Roske, G. J., Some nonlinear multiphase reactions, Stud. Appl. Math., 55 (1976), pp. 231-238.

[41] Rothenberg, J. E., Modulational instability for normal dispersion, Phys. Rev. A, 42 (1990), pp. 682-685.

[42] Rothenberg, J. E., Observation of the buildup of modulational instability from wavebreaking, Opt. Lett., 16 (1991), pp. 18-20.

[43] Rowlands, G. B., On the stability of solutions of the non-linear Schrödinger equation, IMA J. Appl. Math., 13 (1974), pp. 367-377.

[44] Stamper-Kurn, D. M., Andrews, M. R., Chikkatur, A. P., Inouye, S., Miesner, H.-J, Stenger, J. , and Ketterle, W., Optical Confinement of a Bose-Einstein Condensate, Phys. Rev.Lett. 80 (1998), pp. 2027.

[45] Thelwell, R. J., Carter, J. D., and Deconinck, B., Instabilities of one-dimensional stationary solutions of the cubic nonlinear Schrödinger equation, J. Phys. A. 39 (2006), pp. 73-84.

[46] Tsurumi, T. S., Morise, H., And Wadati, M., Stability of Bose-Einstein condensates confined in traps, Int. J. Mod. Phys. B. 14 (2000), pp. 655-719.

[47] Wiggins, S., Introduction to Applied Nonlinear Dynamical Systems and Chaos, Texts in Applied Mathematics, vol 2. 2nd edn. Springer, New York, NY, 2003.

[48] Yang, J. And Benney, D. J., Some properties of nonlinear wave systems, Stud. Appl. Math., 96 (1996), pp. 111-139.

[49] Yang, J., Coherent structures in weakly birefringent optical fibers, Stud. Appl. Math., 97 (1996), pp. 127-148.

[50] Zakharov, V. E., Stability of periodic waves of finite amplitude on the surface of a deep fluid, J. Appl. Mech. Tech. Phys. 9 (1968), pp. 190-194.

[51] Zakharov, V. E., Collapse of Langmuir waves, Sov. Phys. JETP. 35 (1972), pp. 908-914. 